\title{
Análise da confiabilidade e concordância dos métodos paquímetro e polpas digitais na mensuração da diástase do músculo reto abdominal
}

\author{
Analysis of reliability and agreement of the methods caliper and \\ fingerbreadths to measure the rectus abdominis diastasis
}

Ana Carolina Rodarti Pitangui ${ }^{1}, 2$, Letícia Kazue Fukagawa1, Carla Santos Barbosa1 ${ }^{1}$, Alaine Souza Lima², Mayra Ruana de Alencar Gomes², Rodrigo Cappato de Araújo ${ }^{1,2,3}$

${ }^{1}$ Curso de Fisioterapia, Universidade de Pernambuco (UPE) - Petrolina (PE), Brasil.

${ }^{2}$ Programa de Pós-Graduação em Hebiatria, UPE - Recife (PE), Brasil.

${ }^{3}$ Programa Associado de Pós-Graduação em Educação Física, UPE/Universidade Federal da Paraíba (UFPB) - Recife (PE), Brasil.

DOI: http://dx.doi.org/10.7322/abcshs.v41i3.904

\begin{abstract}
RESUMO
Introdução: Métodos palpatórios como paquímetro e polpas digitais são técnicas clinicamente viáveis para determinar a diástase dos músculos retos abdominais. Objetivo: Avaliar a confiabilidade e a concordância dos métodos paquímetro e polpas digitais na mensuração da diástase do músculo reto abdominal no puerpério imediato. Métodos: Estudo descritivo do tipo transversal com mulheres no puerpério imediato. As medidas foram realizadas em momentos distintos por duas avaliadoras. Os pontos de referência para mensuração foram: três dedos $(4,5 \mathrm{~cm})$ acima e abaixo da cicatriz umbilical e na região umbilical. A diástase foi graduada por meio do paquímetro e pelo número de dedos entre as bordas mediais dos músculos retos abdominais. Foi estimado um valor de 1,5 cm para cada dedo. Considerou-se presença de diástase quando ocorreu afastamento maior que $3 \mathrm{~cm}$ entre as bordas mediais dos retos abdominais. A análise estatística foi realizada pelo coeficiente de Kappa ponderado, para avaliação da concordância entre as técnicas, e pelo coeficiente de correlação intraclasse para avaliar a confiabilidade. Em todas as análises foi adotado nível de significância $\mathrm{p}<0,05$. Resultados: Foram avaliadas 261 puérperas, com média de idade de $23,74 \pm 6,42$ anos. Destas, $143(54,8 \%)$ eram primíparas e $118(45,2 \%)$ multíparas. Foram verificados valores excelentes na confiabilidade da técnica das polpas digitais e valores moderados com o uso do paquímetro. A concordância entre as duas técnicas demonstrou-se excelente. Conclusão: As duas técnicas, polpas digitais e paquímetro, revelaram-se instrumentos confiáveis e com concordância na mensuração da diástase do músculo reto abdominal.
\end{abstract}

Palavras-chave: período pós-parto; reprodutibilidade dos testes; abdome; fisioterapia; paridade.

\begin{abstract}
Introduction: Palpatory methods such as caliper and fingerbreadths are clinically viable techniques to determine the rectus abdominis diastasis. Objective: To evaluate the reliability and agreement of the fingerbreadths and caliper methods on measuring the rectus abdominis diastasis in the immediate postpartum. Methods: A descriptive crosssectional study with immediate postpartum women. The measurements were performed at different times by two evaluators. The reference points for measurement were: three fingers $(4.5 \mathrm{~cm})$ above and below the umbilicus region and at the umbilicus. The diastasis was scored by the caliper and by the number of fingers between the medial edges of the rectus abdominal muscles. A value of $1.5 \mathrm{~cm}$ was estimated for each finger. The diastasis was considered present when a distance greater than $3 \mathrm{~cm}$ occurred between the medial edges of the rectus abdominal. Statistical analysis was performed using weighted Kappa coefficient, to assess the agreement between the techniques, and intra-class correlation coefficient to assess reliability. In all analyses, a significance level of $p<0.05$ was adopted. Results: A total of 261 postpartum women were evaluated, with mean age of $23.74 \pm 6.42$ years. Of these, $143(54.8 \%)$ were primiparous and 118 $(45.2 \%)$ were multiparous. Excellent values of reliability were verified in the fingerbreadths technique and moderated ones were observed with the use of caliper. The agreement between the two techniques demonstrated to be excellent. Conclusion: Both techniques, fingerbreadths and caliper, have proven to be reliable instruments with agreement in measuring the rectus abdominis diastasis.
\end{abstract}

Keywords: postpartum period; reproducibility of results; abdomen; physical therapy specialty; parity. 


\section{INTRODUÇÃO}

Durante a gravidez e o pós-parto imediato, é comum o desenvolvimento da diástase dos músculos retos abdominais (DMRA) ${ }^{1-3}$, condição caracterizada pelo afastamento desses músculos na linha média, a linha alba ${ }^{4-7}$, e que pode causar consequências negativas para a saúde ${ }^{1}$. Esse afastamento decorre da ação de hormônios como a relaxina, o estrógeno e a progesterona ${ }^{8}$ sobre o tecido conectivo e do estresse mecânico na parede abdominal ocasionado pelo crescimento do feto ${ }^{2,3,8,9}$.

Os músculos abdominais desempenham importantes funções na postura e na estabilidade do tronco e da pelve ${ }^{10}$, e a presença de DMRA pode resultar em desvantagem biomecânica para esses músculos ${ }^{11}$. Essa condição, associada ao relaxamento ligamentar próprio da gestação, predispõe a puérpera a desenvolver lombalgia, além de aumentar o risco de tensão lombossacral ${ }^{12}$.

A DMRA deve ser avaliada acima ou abaixo do nível umbilical, bem como no mesmo nível ${ }^{7,13}$. Como critério diagnóstico, consideram-se valores de afastamento dos músculos retos abdominais com mais de $3 \mathrm{~cm}$ de largura como acima dos padrões de normalidade ${ }^{14}$, contudo ainda não há consenso sobre esse valor de referência, podendo variar de 2 a $3 \mathrm{~cm}^{3,9}$. Na maioria dos casos, é possível avaliar a DMRA facilmente obtendo-se o diagnóstico por meio da história clínica e do exame físico, já que os músculos retos abdominais se encontram posicionados superficialmente ${ }^{15}$.

A viabilidade clínica de uma ferramenta é importante na escolha de um método de medição. Métodos palpatórios como paquímetro e polpas digitais são técnicas clinicamente viáveis para determinar a DMRA ${ }^{1}$.

A técnica de mensuração mais utilizada e de fácil execução é a das polpas digitais entre as bordas mediais dos músculos retos abdominais ${ }^{4,7}$. Embora seja bastante empregada, alguns autores a consideram questionável, por conta da variação anatômica dos dedos dos avaliadores ${ }^{2,8}$. O paquímetro é um medidor preciso de diâmetros e espessuras, de uso comum na engenharia e de avaliação fidedigna no fornecimento de medidas objetivas ${ }^{12,16,17}$, entretanto certos autores acreditam que a confiabilidade de ambas as técnicas ainda não é muito clara ${ }^{18}$.

Em decorrência da importância de ter instrumentos confiáveis e de fácil aplicabilidade para subsidiar a prática clínica do profissional que atua em saúde da mulher, acrescida à escassez de estudos que se propuseram a avaliar a confiabilidade de ambas as técnicas, justifica-se a realização do presente estudo, que teve como objetivo avaliar a confiabilidade interexaminadores e a concordância dos métodos paquímetro e polpas digitais na mensuração da DMRA de mulheres durante o puerpério imediato.

\section{MÉTODOS}

Trata-se de um estudo descritivo do tipo transversal desenvolvido no período de agosto a novembro de 2012 com puérperas que se encontravam no alojamento conjunto do Hospital
Dom Malan, situado na cidade de Petrolina, Pernambuco, sob gestão do Instituto de Medicina Integral Professor Fernando Figueira (IMIP).

O presente estudo foi aprovado pelo Comitê de Ética em Pesquisa da Universidade de Pernambuco, sob Certificado de Apresentação para Apreciação Ética (CAAE) n. ${ }^{\circ}$ 02637712.0.0000.5207, e todas as voluntárias assinaram o termo de consentimento livre e esclarecido (TCLE), segundo a Resolução n. ${ }^{\circ} 466 / 12$ do Conselho Nacional da Saúde (CNS).

Foram incluídos os dados de puérperas primíparas e multíparas submetidas exclusivamente ao parto vaginal — respeitando-se um período de descanso de seis horas pós-parto - que falassem e entendessem a língua portuguesa e estivessem com as informações do prontuário completas. Foram excluídas as puérperas submetidas a cesárea anterior ou parto múltiplo, que apresentavam obesidade e que possuíam quaisquer patologias associadas à gravidez, como polidrâmnio, macrossomia fetal e desordens hipertensivas da gestação, previamente diagnosticadas pela equipe médica na gestação atual e/ou anterior.

Os dados das puérperas foram coletados por duas examinadoras devidamente treinadas. Inicialmente, foi realizada consulta aos prontuários e, em seguida, foram abordadas as mulheres que atendiam aos critérios de inclusão. Antes de iniciar-se a coleta de dados, as puérperas foram esclarecidas quanto aos objetivos e procedimentos do estudo. Os dados dos prontuários foram transcritos para uma ficha de avaliação, previamente elaborada pelos examinadores.

Nessa ficha, foram registradas informações como idade, estado civil, etnia, escolaridade, profissão, massa, estatura, índice de massa corporal (IMC), antecedentes obstétricos, incluindo paridade, idade gestacional (IG), presença de episiotomia e/ou lacerações do períneo e intercorrências obstétricas e puerperais, além das informações do recém-nascido, como sexo, massa e estatura corporal.

Ao término do preenchimento, a examinadora 1 abordava a paciente e realizava as mensurações nos músculos retos abdominais. Posteriormente, em outro período do dia, a examinadora 2 realizava os mesmos procedimentos na paciente.

O teste da DMRA foi feito nos níveis supraumbilical, umbilical e infraumbilical por meio de duas técnicas: polpas digitais, graduadas conforme o número de polpas colocadas entre as bordas mediais do músculo reto abdominal da puérpera; e paquímetro.

Os pontos de referência para mensuração da DMRA foram três dedos $(4,5 \mathrm{~cm})$ acima e abaixo da cicatriz umbilical e na região umbilical ${ }^{3,7}$. Estipulou-se o valor de $1,5 \mathrm{~cm}$ para cada dedo. Considerou-se presença de DMRA quando ocorreu afastamento maior que $3 \mathrm{~cm}$ entre as bordas mediais dos retos abdominais ${ }^{14}$.

A mensuração aconteceu com a puérpera no leito, na posição supina. Com o quadril fletido e os joelhos flexionados a $90^{\circ}$, os pés ficavam apoiados no leito, e os membros superiores, estendidos ao longo do corpo. Solicitou-se, nessa posição, a flexão 
anterior do tronco até que as espinhas das escápulas ficassem fora do leito ${ }^{19}$. No momento em que a puérpera realizava a flexão anterior do tronco, a avaliadora mensurava o afastamento dos músculos retos abdominais.

Inicialmente, foi realizada a medida pelo método das polpas digitais: o avaliador inseria os dedos perpendicularmente entre as bordas mediais dos músculos retos abdominais. Em seguida, a paciente repetia o mesmo movimento, e marcava-se a separação dos retos abdominais com um lápis dermatográfico para posterior mensuração com o paquímetro. Utilizou-se o paquímetro de $60 \mathrm{~cm}$ da marca Cescorf ${ }^{\boxplus}$, com comprimento de $550 \mathrm{~mm}$ úteis. Após a finalização das medições, a marcação do lápis foi apagada com um algodão embebido em álcool, para que não houvesse indução na medição pela examinadora 2 .

Durante as avaliações, apenas um examinador permaneceu com a voluntária, evitando assim a possível troca ou dedução de informações nos dados coletados. Os métodos empregados na avaliação da DMRA podem ser vistos na Figura 1.

Para a quantificação do número total de sujeitos, foi empregado o programa WinPepi, considerando a estatística bilateral $\alpha=0,05$ e poder de $95 \%$. Com a estimativa cedida pelo hospital da realização de 300 partos normais por mês, foram levadas em conta como a população total no período de três meses de coleta 900 puérperas, com proporção estimada de DMRA supraumbilical de $76,6 \%^{19}$, precisão absoluta de $5 \%$ e perda de $20 \%$, sendo sugerida uma amostra de 264 puérperas.
Os dados foram processados e analisados pelo programa Statistical Package for the Social Sciences (SPSS), versão 20. A análise estatística foi realizada por meio do índice Kappa ponderado para a avaliação da concordância entre as técnicas, sendo considerado: de 0,00 a 0,20 pobre; 0,21 a 0,40 razoável; 0,41 a 0,60 boa; 0,61 a 0,80 muito boa; e 0,81 a 1,0 excelente ${ }^{20}$. O coeficiente de correlação intraclasse (CCI) foi empregado para avaliar a confiabilidade interexaminadores, com os seguintes valores: pobre $(<0,4)$, moderada $(0,4-0,75)$ e excelente $(>0,75)^{21}$ Em todas as análises, foi adotado nível de significância $\mathrm{p}<0,05$.

\section{RESULTADOS}

A amostra foi constituída por 261 puérperas com média de idade de 23,74 $\pm 6,42$ anos. Destas, 143 (54,8\%) eram primíparas e $118(45,2 \%)$ multíparas. A Tabela 1 exibe os dados referentes à confiabilidade interexaminadores nas diferentes técnicas de mensuração da DMRA. Como se pode observar, em todas as regiões avaliadas foram verificados valores excelentes de CCI para a técnica das polpas digitais e valores moderados para o paquímetro.

Na Tabela 2, visualizam-se as frequências absoluta e relativa da DMRA nas regiões supraumbilical, umbilical e infraumbilical e a concordância do índice Kappa ponderado entre as duas técnicas (polpas digitais e paquímetro). Os valores foram excelentes em quase todas as análises, com exceção da região supraumbilical, considerada muito boa na avaliação do examinador 1 .

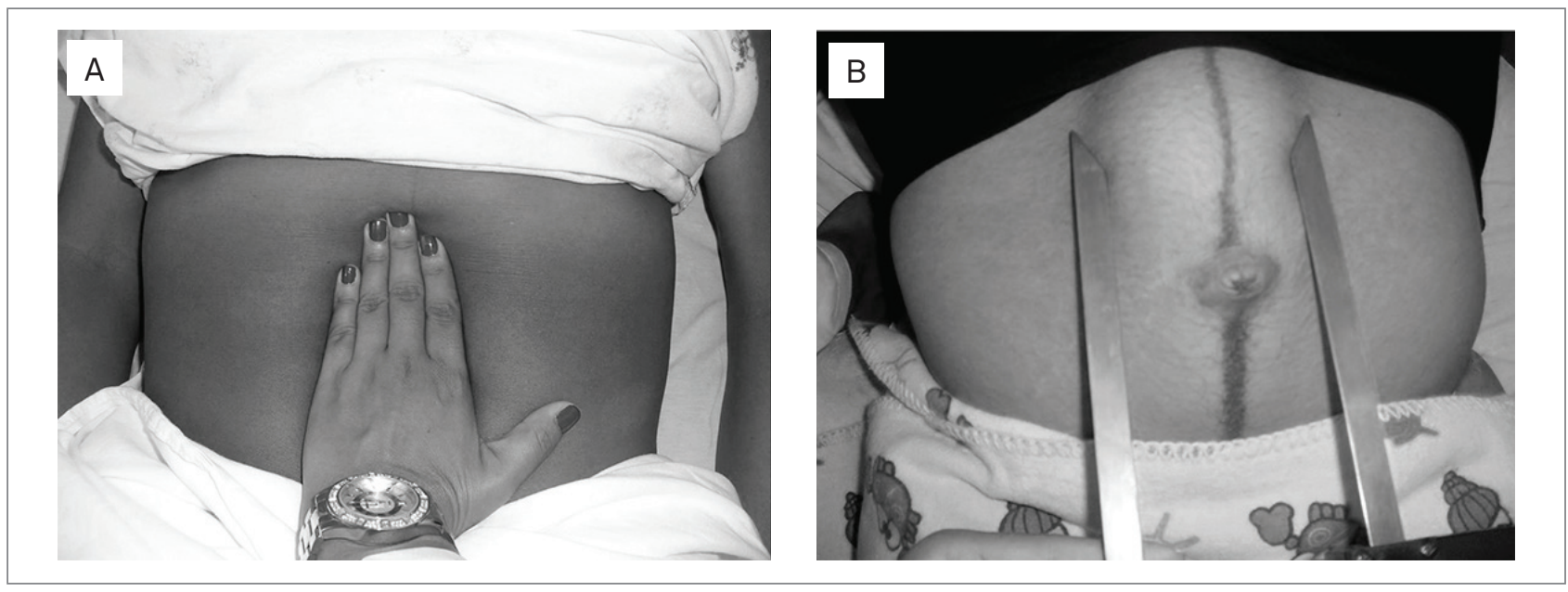

Figura 1: Mensuração da diástase dos músculos retos abdominais no nível supraumbilical (A) técnica das polpas digitais e (B) paquímetro

Tabela 1: Valores de confiabilidade interexaminadores verificados nas técnicas de polpa digital e paquímetro

\begin{tabular}{|l|c|c|c|c|c|}
\hline Técnica/região umbilical & $\begin{array}{c}\text { Examinador } \mathbf{1} \\
\text { Média (DP) }\end{array}$ & $\begin{array}{c}\text { Examinador } \mathbf{2} \\
\text { Média (DP) }\end{array}$ & EPM & $\mathbf{C C l}^{\mathbf{I}}$ & $\mathbf{\mathbf { C } _ { 9 5 \% }}$ \\
\hline Polpa digital supraumbilical & $4,15(1,39)$ & $4,24(1,34)$ & 0,05 & 0,88 & $0,85-0,91$ \\
\hline Polpa digital umbilical & $3,36(1,19)$ & $3,44(1,11)$ & 0,05 & 0,86 & $0,82-0,89$ \\
\hline Polpa digital infraumbilical & $2,01(1,08)$ & $2,20(1,20)$ & 0,05 & 0,86 & $0,82-0,89$ \\
\hline Paquímetro supraumbilical & $2,87(3,29)$ & $2,78(1,92)$ & 0,17 & 0,64 & $0,54-0,71$ \\
\hline Paquímetro umbilical & $1,82(1,81)$ & $2,14(3,04)$ & 0,15 & 0,64 & $0,54-0,72$ \\
\hline Paquímetro infraumbilical & $0,58(1,19)$ & $0,69(1,41)$ & 0,07 & 0,74 & $0,69-0,81$ \\
\hline
\end{tabular}

EPM: erro padrão da medida; CCl: coeficiente de correlação intraclasse; IC: intervalo de confiança; DP: desvio padrão; $p<0,05$. 
Tabela 2: Análise da concordância entre as técnicas da polpa digital e paquímetro

\begin{tabular}{|c|c|c|c|c|c|c|}
\hline \multirow{3}{*}{$\begin{array}{l}\text { Região umbilical } \\
\text { Classificação }\end{array}$} & \multicolumn{3}{|c|}{ Examinador 1} & \multicolumn{3}{|c|}{ Examinador 2} \\
\hline & Polpa digital & Paquímetro & Kappa & Polpa digital & Paquímetro & Kappa \\
\hline & n (\%) & n (\%) & $I C_{95 \%}$ & n (\%) & n (\%) & $I C_{95 \%}$ \\
\hline \multicolumn{7}{|l|}{ Supraumbilical } \\
\hline Normal & $98(37,5)$ & $122(46,7)$ & 0,80 & $82(31,4)$ & $91(34,9)$ & 0,91 \\
\hline Diástase & $163(62,5)$ & $139(53,3)$ & $0,72-0,87$ & $179(68,6)$ & $170(65,1)$ & $0,85-0,96$ \\
\hline \multicolumn{7}{|l|}{ Umbilical } \\
\hline Normal & $154(59,0)$ & $171(65,5)$ & 0,86 & $127(48,7)$ & $137(52,5)$ & 0,89 \\
\hline Diástase & $107(41,0)$ & $90(34,5)$ & $0,80-0,93$ & $134(51,3)$ & $124(47,5)$ & $0,84-0,95$ \\
\hline \multicolumn{7}{|l|}{ Infraumbilical } \\
\hline Normal & $236(90,4)$ & $238(91,2)$ & 0,93 & $218(83,5)$ & $220(84,3)$ & 0,92 \\
\hline Diástase & $25(9,6)$ & $23(8,8)$ & $0,85-1,00$ & $43(16,5)$ & $41(15,7)$ & $0,85-0,98$ \\
\hline
\end{tabular}

IC: intervalo de confiança; $\mathrm{p} \leq 0,05$.

\section{DISCUSSÃO}

No presente estudo, observou-se que ambas as técnicas, polpas digitais e paquímetro, se revelaram instrumentos confiáveis para classificar a DMRA, sendo encontrados valores superiores de confiabilidade na técnica das polpas digitais.

Desse modo, acredita-se que os maiores valores da técnica das polpas digitais, quando comparados aos do paquímetro, possam ser decorrentes de aspectos relacionados ao procedimento da mensuração, uma vez que o posicionamento e o manejo do paquímetro podem gerar mais dificuldade na execução da medida pelo examinador.

A técnica das polpas digitais é uma ferramenta bastante utilizada para a mensuração da DMRA na prática clínica ${ }^{4}$, sendo considerada de grande utilidade para sua avaliação ${ }^{22,23}$. Em contrapartida, alguns autores não respaldam sua indicação pelo fato de as polpas digitais se basearem em parâmetros subjetivos e tidos como não reprodutíveis, por conta das possíveis variações de largura dos dedos de diferentes terapeutas ${ }^{2,8}$.

O paquímetro é um método pouco oneroso e mais objetivo ${ }^{16,17}$ que apresenta alta confiabilidade intra-avaliador na medição da DMRA em puérperas, tanto em repouso quanto durante a contração do abdômen ${ }^{12}$.

Quanto aos dados da concordância entre as técnicas, observou-se que ambas as ferramentas apresentaram valores excelentes, ou seja, os dois métodos podem ser indicados para a prática clínica, corroborando os achados de outros autores ${ }^{24}$.

Assim, os resultados deste estudo demostram que, além do paquímetro, a técnica das polpas digitais também é um método que pode ser considerado reprodutível e confiável, indo ao encontro de outros autores que respaldam seu potencial na triagem da presença de DMRA ${ }^{24}$.

Contudo ainda são escassos os estudos que avaliaram a confiabilidade interexaminadores da técnica das polpas digitais. Estudo recente desenvolvido por Mota et al. ${ }^{18}$ comparou a confiabilidade da técnica das polpas digitais com a do ultrassom e concluiu que o método das polpas digitais apresenta boa confiabilidade intraexaminador e confiabilidade moderada interexaminador para avaliar a distância inter-retos.

Em recente revisão sistemática sobre métodos de avaliação da DMRA, os autores inferem que, apesar de a técnica das polpas digitais ser amplamente utilizada clinicamente, ela tem sido subavaliada. Todavia, alguns dados de confiabilidade podem mostrar que o método é suficiente para o reteste (um avaliador) e, potencialmente, para o rastreio da presença de DMRA. Determinar se esse afastamento está acima ou abaixo de um valor de corte, com coerência entre avaliadores na classificação e categorização dos pacientes, é um dado importante em termos clínicos ${ }^{24}$.

Soma-se ainda positivamente aos achados deste estudo o fato de a técnica das polpas digitais ser de fácil emprego e manuseio, baixo custo e não depender de um instrumento externo de avaliação, condições que favorecem seu uso em locais desprovidos de outros instrumentos de avaliação, visto que sua aplicação não atrapalharia a exatidão do diagnóstico da DMRA ${ }^{18}$.

Desse modo, torna-se inadmissível ao fisioterapeuta não acrescentar à avaliação de mulheres que se encontram no ciclo gravídico-puerperal dados referentes à mensuração da DMRA.

Entretanto cabe ressaltar que outras técnicas de medida são descritas pela literatura para a avaliação da DMRA, como exames de imagem. A ultrassonografia é uma técnica de custo relativo e não invasiva, no entanto sua realização exige treinamento criterioso do avaliador. Além disso, a qualidade da imagem pode ser prejudicada pela interposição de gordura e fibrose ${ }^{15}$. A tomografia computadorizada é um bom método para avaliar a posição dos músculos retos abdominais, mas é dispendiosa e expõe o paciente à radiação ${ }^{15,17}$. De qualquer modo, ainda não existe evidência suficiente de que a tomografia computadorizada possa ser considerada padrão ouro, como é frequentemente afirmado ${ }^{24}$.

Em função dessas condições, ambas as técnicas se tornam de difícil acesso ao profissional que atua na prática clínica, justificando assim a importância do presente estudo, como suporte para a indicação de ferramentas confiáveis que devem ser incorporadas à rotina do profissional. 
Algumas limitações devem ser mencionadas em relação a este estudo, como a não realização da avaliação de confiabilidade intraexaminadores. Porém, em razão de a alta hospitalar das puérperas ter ocorrido em um breve período, não foi possível reavaliá-la em outro dia.

Outro fato diz respeito ao teste da DMRA, que foi feito apenas com os músculos retos abdominais em contração, sendo indicada em estudos futuros a realização do teste com o músculo relaxado. Em repouso, o tamanho da DMRA pode apresentar correlação linear positiva significativa com a largura da medição da diástase ativa, obtendo resultados maiores na separação, quando comparado ao músculo contraído ${ }^{12}$.

Ademais, é possível que o excesso de gordura abdominal seja um fator importante que interfira na confiabilidade da mensuração da DMRA, uma vez que, no decorrer do teste, pode ocorrer dificuldade em definir suas bordas mediais pela redução do tônus muscular, flacidez abdominal e grande espessura da gordura subcutânea ${ }^{12}$.

Diante do exposto, vale ainda ressaltar que a ineficiência dos músculos abdominais pode gerar impacto negativo na autoestima e na saúde das mulheres durante e após a gestação ${ }^{10}$, além de ocasionar, em médio e longo prazo, redução na qualidade de vida ${ }^{17}$. Repercussões na estabilização do tronco e da postura, queixas de lombalgia ${ }^{3,7,12,23}$ e fraqueza dos músculos do assoalho pélvico ${ }^{25}$ têm sido reportadas pela literatura. Postula-se que haja relação entre a presença da DMRA e o diagnóstico de incontinência urinária por esforço, incontinência fecal e prolapso de órgãos pélvicos ${ }^{23}$.
Desse modo, a detecção precoce da DMRA é de grande importância para a prática clínica, uma vez que, baseado nesse diagnóstico, o profissional poderá propor condutas adequadas. Estudos sugerem que exercícios físicos antes e depois da gestação, com o intuito de fortalecer a musculatura abdominal/core $e^{1,10,23,25,26}$, com fortalecimento de forma isométrica ${ }^{27}$, bem como educação e cuidados com a postura, exercícios aeróbios e suportes externos (cintas abdominais), podem prevenir e/ou recuperar a DMRA ${ }^{25,26}$.

Além disso, ainda não está claro qual é o tipo de intervenção não cirúrgica mais apropriado para evitar e/ou reduzir a DMRA. Os poucos estudos realizados apresentam qualidade insuficiente de evidência para recomendarem alguma conduta específica ${ }^{10}$.

Nesse sentido, sugere-se o desenvolvimento de estudos futuros que tentem elucidar as lacunas acerca da DMRA, propondo a análise da confiabilidade dessas técnicas com outros instrumentos, como o ultrassom, que desenvolvam outros tipos de delineamento, como o clínico, e promovam o acompanhamento de grupos distintos com protocolos compostos de diferentes condutas, com o intuito de determinar o melhor manejo a ser adotado.

Por fim, pode-se concluir, com o presente estudo, que ambas as técnicas avaliadas, polpas digitais e paquímetro, se revelaram instrumentos com confiabilidade e concordância. O emprego desses métodos fornece uma abordagem de avaliação composta de medidas precisas, com parâmetros fidedignos e reprodutíveis. Espera-se que os resultados deste trabalho possam contribuir para respaldar e incentivar o uso de tais instrumentos na prática clínica dos profissionais que atuam em instituições de assistência ao parto e pós-parto.

\section{REFERÊNCIAS}

1. Keeler J, Albrecht M, Eberhardt L, Horn L, Donnelly C, Lowe D. Diastasis recti abdominis: a survey of women's health specialists for current physical therapy clinical practice for postpartum women. J Women's Health Phys Therap. 2012;36(3):131-42. http://dx.doi.org/10.1097/JWH.0b013e318276f35f

2. Bursch SG. Interrater reliability of diastasis recti abdominis measurement. Phys Ther. 1987;67(7):1077-9.

3. Boissonnault JS, Blaschak MJ. Incidence of diastasis recti abdominis during the childbearing year. Phys Ther. 1988;68(7):1082-6.

4. Hsia M, Jones S. Natural resolution of rectus abdominis diastasis. Two single case studies. Aust J Physiother. 2000;46(4):301-7.

5. Hickey F, Finch JG, Khanna A. A systematic review on the outcomes of correction of diastasis of the recti. Hernia. 2011;15(6):607-14. http://dx.doi.org/10.1007/s10029-011-0839-4

6. Liaw LJ, Hsu MJ, Liao CF, Liu MF, Hsu AT. The relationships between inter-recti distance measured by ultrasound imaging and abdominal muscle function in postpartum women: a 6-month follow-up study. J Orthop Sports Phys Ther. 2011;41(6):435-43 http://dx.doi.org/10.2519/jospt.2011.3507

7. Chiarello CM, McAuley JA. Concurrent validity of calipers and ultrasound imaging to measure interrecti distance. J Orthop Sports Phys Ther. 2013;43(7):495-503.

http://dx.doi.org/10.2519/jospt.2013.4449
8. Rett MT, Araújo FR, Rocha I, Silva RA. Diástase dos músculos retoabdominais no puerpério imediato de primíparas e multíparas após o parto vaginal. Fisioter Pesq. 2012;19(3):236-41.

http://dx.doi.org/10.1590/S1809-29502012000300008

9. Coldron Y, Stokes MJ, Newham DJ, Cook K. Postpartum characteristics of rectus abdominis on ultrasound imaging. Man Ther. 2008:13(2):112-21.

http://dx.doi.org/10.1016/j.math.2006.10.001

10. Benjamin DR, van de Water ATM, Peiris CL. Effects of exercise on diastasis of the rectus abdominis muscle in the antenatal and postnatal periods: a systematic review. Physiotherapy. 2014; $100(1): 1-8$

http://dx.doi.org/10.1016/j.physio.2013.08.005

11. Gilleard WL, Brown JM. Structure and function of the abdominal muscles in primigravid subjects during pregnancy and the immediate postbirth period. Phys Ther. 1996;76(7):750-62.

12. Boxer S, Jones S. Intra-rater reliability of rectus abdominis diastasis measurement using dial calipers. Aust J Physiother. 1997;43(2):109-14.

13. Baracho E, Baracho SM, Felissíssimo M. Atuação do fisioterapeuta no puerpério imediato e tardio. In: Baracho E. Fisioterapia aplicada à obstetrícia, uroginecologia e aspectos de mastologia. 4th ed. Rio de Janeiro: Guanabara Koogan 2007; p. 241-50. 
14. Noble E. Essential exercises for the childbearing year: a guide to health and comfort before and after your baby is born. 2nd ed. Boston: Houghton Mifflin; 1982; p. 117-23.

15. Mendes DA, Nahas FX, Veiga DF, Mendes FV, Figueiras RG, Gomes $\mathrm{HC}$, et al. Ultrasonography for measuring rectus abdominis muscles diastasis. Acta Cir Bras. 2007;22(3):182-6. http://dx.doi.org/10.1590/S0102-86502007000300005

16. Mesquita LA, Machado AV, Andrade AV. Fisioterapia para redução da diástase dos músculos retos abdominais no pós-parto. Rev Bras Ginecol Obstet. 1999;21(5):267-72. http://dx.doi.org/10.1590/S0100-72031999000500004

17. Barbosa S, de Sá RA, Coca Velarde LG. Diastasis of rectus abdominis in the immediate puerperium: correlation between imaging diagnosis and clinical examination. Arch Gynecol Obstet. 2013;288(2):299-303.

http://dx.doi.org/10.1007/s00404-013-2725-z

18. Mota P, Pascoal AG, Sancho F, Carita AI, Bø K. Reliability of the interrectus distance measured by palpation. Comparison of palpation and ultrasound measurements. Man Ther. 2013;18(4):294-8. http://dx.doi.org/10.1016/j.math.2012.10.013

19. Rett MT, Almeida TV, Mendonça ACR, De Santana JM, Ferreira APL, Araújo KCGM. Fatores materno-infantis associados à diástase dos músculos retos do abdome no puerpério imediato. Rev Bras Saúde Mater Infant. 2014;14(1):73-80. http://dx.doi.org/10.1590/S1519-38292014000100007

20. Weir JP. Quantifying test-retest reliability using the intraclass correlation coefficient and the SEM. J Strength Cond Res. 2005;19(1):231-40. http://dx.doi.org/10.1519/15184.1
21. Fleiss JL. Design and analysis of clinical experiments. 2nd ed. New York: John Wiley \& Sons; 1999.

22. Rett MT, Braga MD, Bernardes NO, Andrade SC. Prevalence of diastasis of the rectus abdominis muscles immediately postpartum: comparison between primiparae and multiparae. Rev Bras Fisioter. 2009;13(4):275-80.

http://dx.doi.org/10.1590/S1413-35552009005000037

23. Turan V, Colluoglu C, Turkyilmaz E, Korucuoglu U. Prevalence of diastasis recti abdominis in the population of young multiparous adults in Turkey. Ginekol Pol. 2011;82(11):817-21.

24. van de Water AT, Benjamin DR. Measurement methods to assess diastasis of the rectus abdominis muscle (DRAM): a systematic review of their measurement properties and meta-analytic reliability generalisation. Man Ther. 2016;21:41-53. http://dx.doi.org/10.1016/j.math.2015.09.013

25. Spitznagle TM, Leong FC, Van Dillen LR. Prevalence of diastasis recti abdominis in a urogynecological patient population. Int Urogynecol J Pelvic Floor Dysfunct. 2007;18(3):321-8. http://dx.doi.org/10.1007/s00192-006-0143-5

26. Sheppard S. The role of transversus abdominus in post partum correction of gross divarication recti. Man Ther. 1996;1(4):214-6.

http://dx.doi.org/10.1054/math.1996.0272

27. Pascoal AG, Dionisio S, Cordeiro F, Mota P. Inter-rectus distance in postpartum women can be reduced by isometric contraction of the abdominal muscles: a preliminary case-control study. Physiotherapy. 2014;100(4):344-8. http://dx.doi.org/10.1016/j.physio.2013.11.006 